\title{
The Uniformal Metallization of the AlSiC Metal Matrix Composite Material Surface
}

\author{
Nikolai Vladimirovich Sevostyanov1, Konstantin Nikolaevich Nishchev², \\ Mikhail Il'ich Novopoltsev² \\ ${ }^{1}$ All-Russian Scientific Research Institute of Aviation Materials, State Research Center (VIAM), Moscow, Russia \\ ${ }^{2}$ Ogarev Mordovia State University, Saransk, Russia \\ Email: nishchev@inbox.ru
}

Received 25 May 2014; revised 23 June 2014; accepted 11 July 2014

Copyright (C) 2014 by authors and Scientific Research Publishing Inc.

This work is licensed under the Creative Commons Attribution International License (CC BY). http://creativecommons.org/licenses/by/4.0/

c) (7) Open Access

\begin{abstract}
The surface of the AISiC composite material consists of aluminum and silicon carbide areas. The uniformal metallization by nickel is necessary in order to provide solderability and high thermal conductivity of the surface. The process of chemical deposition of nickel coatings elaborated in the research center VIAM yields the uniformal highly adhesive metallization. The kinetics of the deposition process has been studied and the estimation of the coatings quality has been made in comparison with commercially available samples produced elsewhere.
\end{abstract}

\section{Keywords}

Composite, Coating, AlSiC, Nickel

\section{Introduction}

Modern high-power electronic devices are designed for commuting electric currents up to $50 \mathrm{kA}$ and voltages up to $8 \mathrm{kV}$. At present, insulate gate bipolar transistors (IGBT) have most widely spread out of all the varieties of power electronic devices [1] [2].

The IGBT module is made as a multilayer construction (Figure 1) in which the semiconductor crystal (5) is placed on the electric insulating DBC plate (6) (direct bonded copper deposition). The DBC plate made from ceramics that has a low thermal expansion coefficient (CTE) is a good insulator and has a high thermal conductivity that provides the effective heatsink from the crystal.

The IGBT module operation with large values of commuted current leads to high values of the thermal energy release which should effectively and safely for the power module be removed to environments. The energy release is so high, that the use of traditional materials for heatsinks (copper, aluminium, etc.) cannot ensure a reliable 


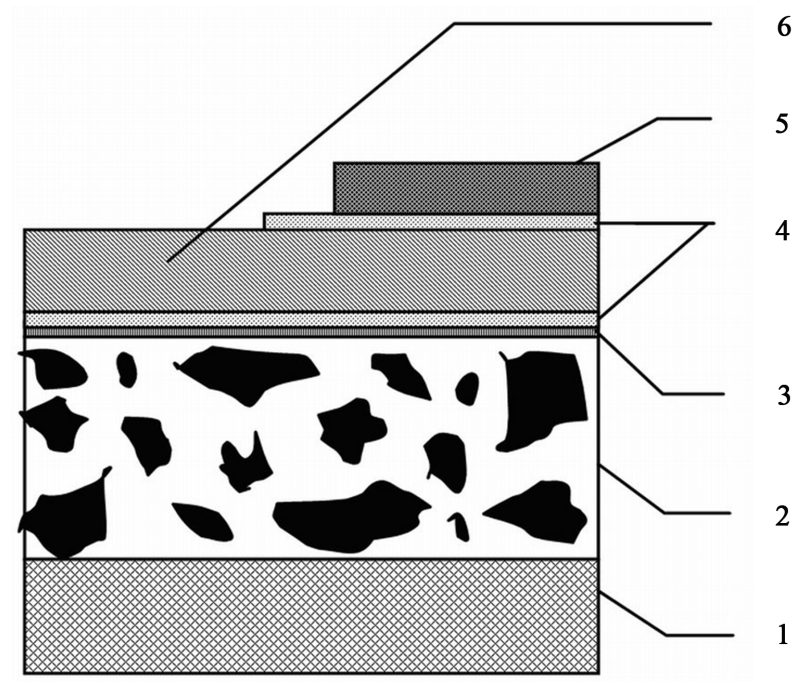

Figure 1. The junction of the soldered semiconductor crystal of the DBC plate with the heatsink base: 1: heatsink, 2: AlSiC base, 3: nickel coating, 4: solder, 5: crystal, 6: DBC plate.

operation of the module at the multiple current commutations due to different CTE elements of the IGBT module and the heatsink, since there arise mechanical tensions which can lead to the module destruction. The metallic composite material (MCM) on the base of the aluminium alloys, reinforced by granules of silicon carbide, possesses all these qualities [2]-[5].

The problem can be solved if use is made of heatsink material with high mechanical strength, high thermal conductivity and the CTE value which is close to that of the DBC module elements. By varying the ratios of Al matrix alloy and $\mathrm{SiC}$ aggregate, one can regulate physical properties of the material and minimize mechanical tensions in the power DBC module. This affords to obtain a high reliability and durable exploitation (not less than 100,000 cycles) of the IGBT modules with the use of the MCM [3] [6] [7].

The DBC plate with a semiconductor crystal is fixed to the heatsink base by tin soldering. As is known, neither aluminium nor silicon carbide possesses solderability by tin solder. Moreover, the uniformal soldering on the whole surface is necessary for the effective heatsink. For this purpose, a uniformal nickel coating is made on the surface of the heatsink plate made of MCM AlSiC.

A simple and inexpensive way of surface metallic coating is the chemical method, but the AlSiC surface is a combination of the silicon carbide and aluminium matrix (Figure 2).

In case of such a heterogeneous surface, uniformal nickel coating by the chemical method is but problematic, problems being related with different ways of preparing surfaces of different materials prior to chemical metallization. Within the traditional method of aluminium chemical nickellization by zincate treatment it is impossible to obtain the nickel coating of silicon carbide areas of the AlSiC MCM, because this treatment does not lead to silicon carbide surface activation and results in metal deposition on aluminium areas only. Methods of dielectrics and semiconductor chemical metallization are known [8]-[10], but these methods require additional ways of treatment, such as sensibilization and activation that complicate the process and cannot be applied to aluminium. The method of aluminium sublayer preliminary coating on the AlSiC surface followed by electrochemical nickel deposition has previously been used [11]. In the research center VIAM has been elaborated technological scheme for chemical nickellization, which allows to exclude intermediate step of coating of the AlSiC surface of aluminium sublayer and to obtain the uniformal AlSiC surface metallization of high adhesive on aluminium and silicon carbide areas.

\section{Material and Experimental Equipment}

The salts and distilled water were used to make electrolytes. The technological process and samples were tested in a 1.5 liter thermostatic glass cell, electrolyte consumption on metal ions being $10 \%$, the concentration of the solution being maintained by well-timed refilling, the laboratory electronic balance AJ-4200 CE being used to 


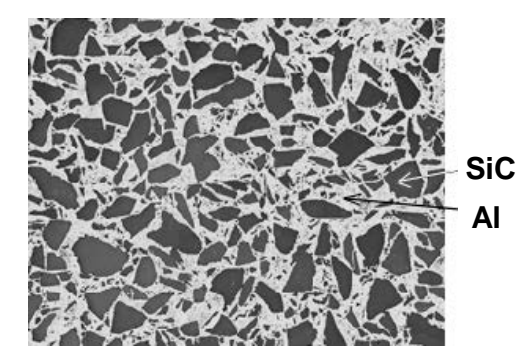

Figure 2. The AlSiC surface macrostructure.

measure the samples and solution components. The area of AlSiC sample deposition was $100 \mathrm{~cm}^{2}$. The X-ray fluorescent surface analyzer Innov-X Alpha Series was used to estimate the quantitative and qualitative composition of the deposited coatings. The control of the nickel coating thickness was made by coating analyzer X-Strata 980, the optical microscope Neophot-21 being used to control the external surface of the coating. The uniformity of the coating was controlled by the scanning microscope JSM-35. Thermocyclic tests were made in a climatic chamber of the type MS-81 Excal 222/TA.

\section{Results and Discussion}

The elaborated technological scheme for chemical nickellization does not require complicated and expensive operations, and the electrolyte used for the purpose possesses an increased stability. The deposited nickel coating contains phosphorus because the electrolyte includes hypophosphite consumption. The phosphorus content is to be varied from $6 \%$ to $12 \%$. The content of phosphorus in the coating increases with an increase in electrolyte consumption, the elementary phosphorus being restored on cathodes and included into the coating as the nickel phosphide type $\mathrm{Ni}_{2} \mathrm{P}$ [12].

Figure 3 shows the average rate of nickel deposition. There are 3 the regions. During the first 3 hours, the deposition rate linearly decreases, which can be related to the consumption of reagents in the solution. Then the linear decrease in the nickel deposition rate sharply comes to a horizontal line. As a result of chemical reactions (nickel deposition, hydrogen release, and phosphorus restoration), the solution accumulates reaction products which slow down the process of nickel deposition. The nickel deposition rate decreases with an increase in active reagent consumption in the solution.

Figure 4 shows the nickel deposition surfaces of the commercial sample (a) and the VIAM sample (b). The sample (a) exhibits a visible roughness of the surface, which is related to technological features of AlSiC manufacturing. A high phosphorus content (up to 16\%) in the nickel coating of this sample indicates the chemical method of surface coating. Due to the uniformity of nickel deposition by the chemical method, the initial pattern of the surface is replicated, practically, with no distortion and silicon carbide crystals are distinctly seen on the matrix alloy surface.

The surface of the VIAM sample was refined up to the roughness $\mathrm{R} \alpha<0.8$ and due to this, the surface pattern is not so distinctly seen. The coating as thick as $3 \mu \mathrm{m}$ can preserve the base structure [13], and due to this, one can observe the nickel coating heterogeneity caused by heterogeneity of aluminium and silicon carbide surfaces.

Figure 5 shows the cross-section fracture of the AlSiC MCM: (a) is the commercial sample, (b) is the VIAM sample.

In Figure 5 of the commercial sample fracture, the nickel layer thickness is seen to be from 5 to $7 \mathrm{mkm}$. The nickel coating on the surface of the VIAM sample, as thick as $2 \mathrm{mkm}$, is continuous and uniformal in the thickness not only on the aluminium, but also on the silicon carbide areas.

The quality of adhesion of sample coatings was tested by way of heating the samples up to $200^{\circ} \mathrm{C}$ followed cooling up to temperatures $-50^{\circ} \mathrm{C}$ in 5 cycles. No cleavage, cracking, and swelling were observed.

\section{Conclusion}

The process of chemical nickellization of AlSiC surfaces elaborated in the VIAM research center affords to obtain a continuous metallic coating uniformal in thickness on heterogeneous areas. At thickness of $2 \mathrm{~m} \cdot \mathrm{km}$, the coating is continuous and homogeneous in the thickness. If necessary, the coating thickness can be enlarged 


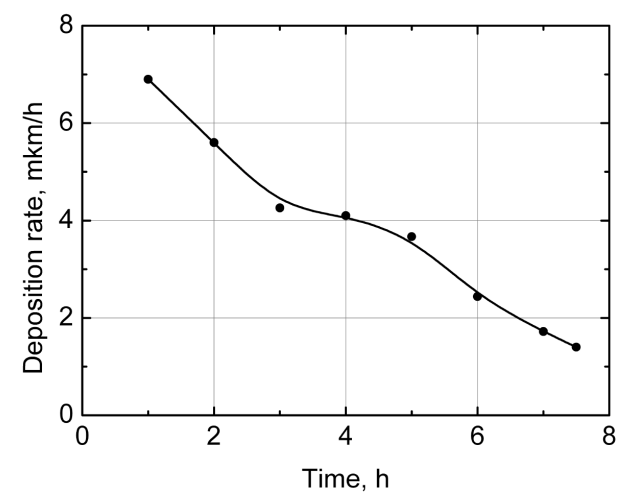

Figure 3. The nickel deposition average rate.

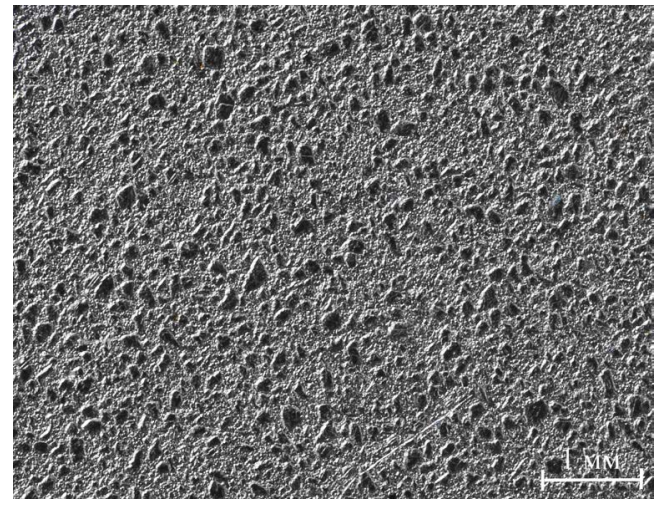

(a)

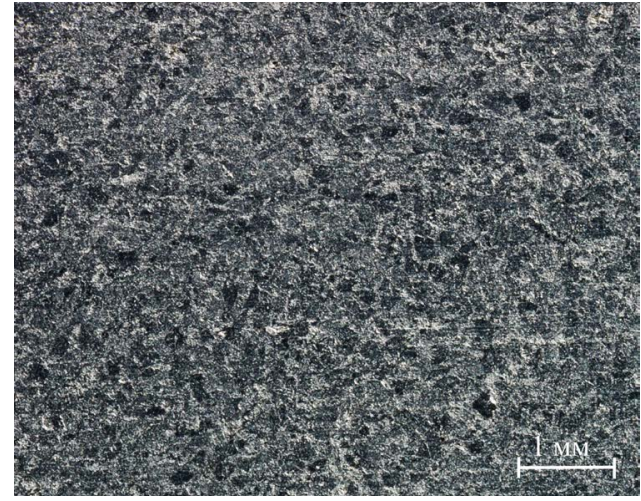

(b)

Figure 4. The nickel deposition surfaces: (a) Of the commercial sample; (b) The VIAM sample.

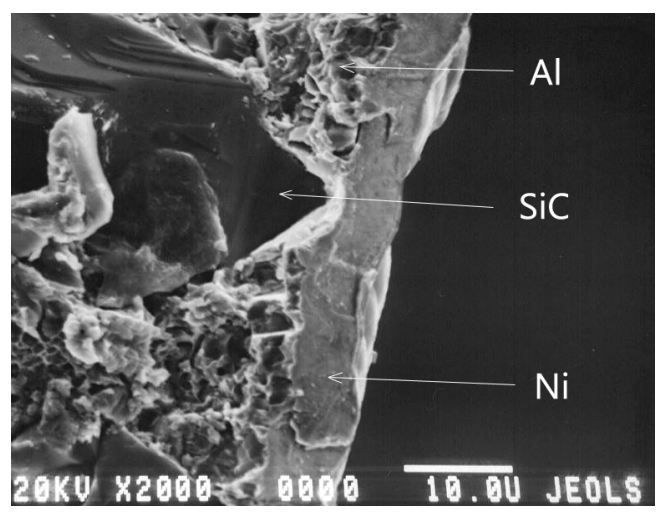

(a)

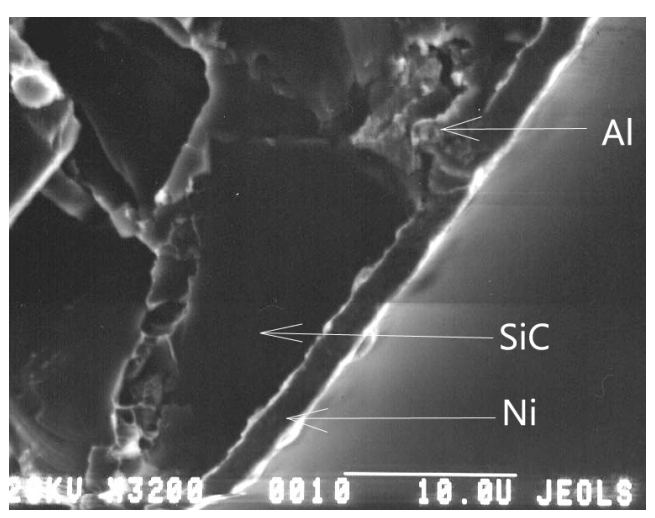

(b)

Figure 5. The cross-section fracture of the AlSiC: (a) The commercial sample; (b) The VIAM sample.

when correcting the time of the process. A required functional galvanic coating, golden being included, can be deposited onto the surface of the layer of chemically deposited nickel coating. Thus, the samples of chemical nickellization by the process elaborated meet technological requirements and are in no way inferior to analogues produced elsewhere.

\section{References}

[1] Sarabjot, S. and Ryssel, H. (2008) Lifetime of Power Modules. 7th Indo-German Winter Academy, Chennai, 13-19 December 2008. http://www.leb.eei.uni-erlangen.de/winterakademie/2008/report/content/course03/pdf/0312.pdf 
[2] Gilleo, K. (2005) MEMS/MOEMS Packaging: Concepts, Designs, Materials, and Processes. McGraw-Hill Nanoscience and Technology Series, Chicago, NY, 239p.

[3] Kablov, E.N., et al. (2011) Properties and Use of Highly Aggregated Metal Matrix Composite Material Al-SiC. Bulletin of N.I. Lobachevsky State University, 3-1, 56-59.

[4] Kablov, E.N., et al. (2012) Manufacturing, Properties and Use of Heatsink Bases from MCM Al-SiC in Power Electronics and Convertor Technology. Aviation Materials and Technologies, 2, 20-23.

[5] Kablov, E.N., et al. (2012) Metal Matrix Composite Material on the Al-SiC. Aviation Materials and Technologies, 5, 373-380.

[6] Kablov, E.N., et al. (2010) Enhancement of Power IGBT Module Reliability with the Use High-Aggregated MCM System Al-SiC. Aviation Materials and Technologies, 4, 3-6.

[7] Kablov, E.N., et al. (2012) Metallic Composite Materials on the Base of Al-SiC for Power Electronics. Mechanics of Composite Materials and Constructions, 18, 359-368.

[8] Nikandrova, L.I. (1971) Chemical Methods of Metal Coatings. Mashinostroenie, Leningrad, 104.

[9] Patent Russian Federation No. 2219284 from 18.05.2002, No. 2350687 from 22.05.2007.

[10] Shakanskas, M. and Vaskalis A. (1985) Chemical Plastic Metallization. Chimiya, Leningrad, 144.

[11] Nishchev, K.N., et al. (2012) The Use of Metal-Matrix Al-SiC Composites in Heat-Spreading Bases of Power Electronic Devices. Polymer Science Series D, Glues and Sealing Materials, 5, 195-198. http://dx.doi.org/10.1134/S1995421212030185

[12] Iliin, V.A. (1977) Dielectrics Metallization. Mashinostroenie, Leningrad, 180.

[13] Gamburg, Y.D. (1997) Electrochemical Crystallization of Metals and Alloys. Yanus-K, Moscow. 
Scientific Research Publishing (SCIRP) is one of the largest Open Access journal publishers. It is currently publishing more than 200 open access, online, peer-reviewed journals covering a wide range of academic disciplines. SCIRP serves the worldwide academic communities and contributes to the progress and application of science with its publication.

Other selected journals from SCIRP are listed as below. Submit your manuscript to us via either submit@scirp.org or Online Submission Portal.
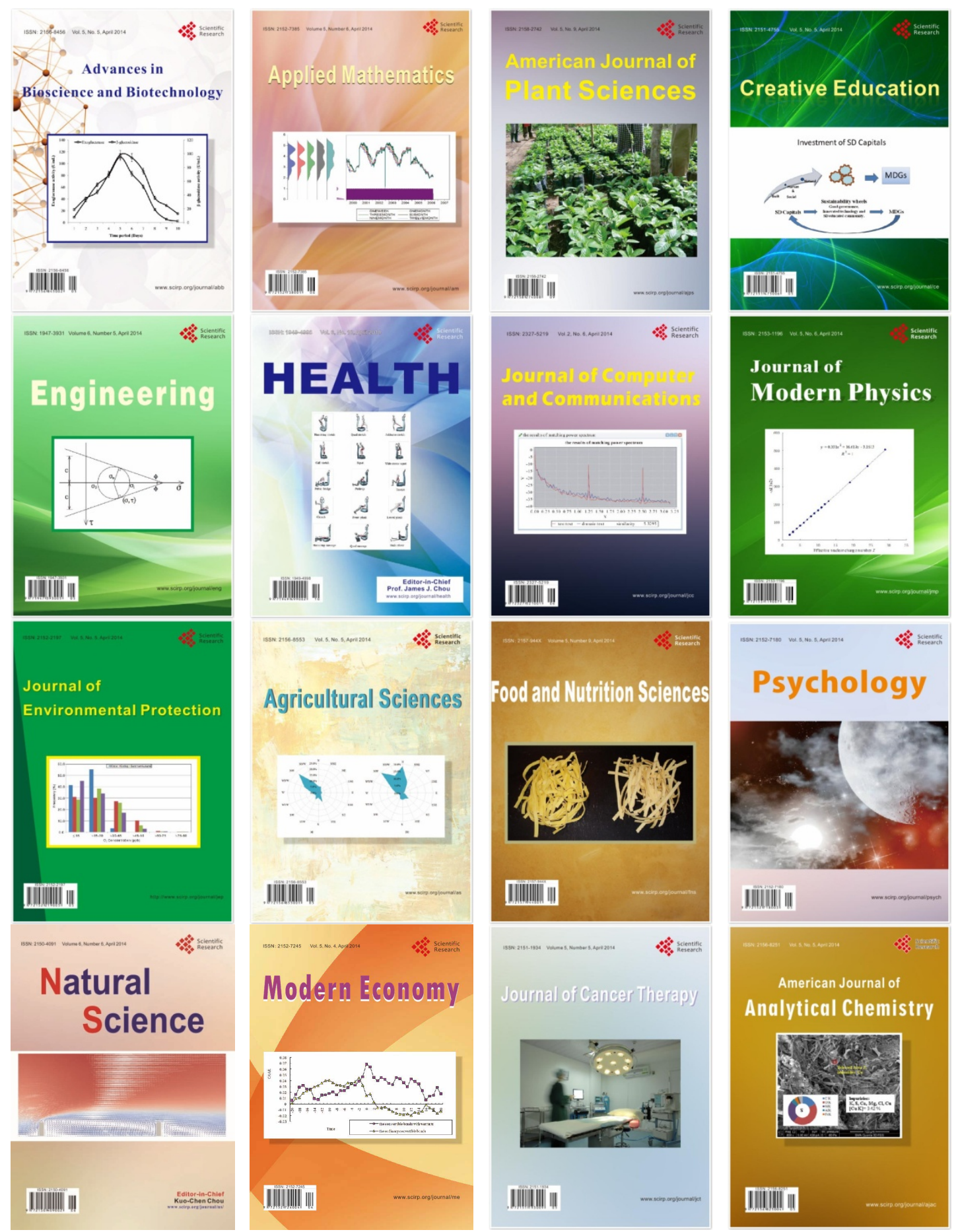